\title{
A NONSTATIONARY PROBLEM IN THE THEORY OF ELECTROLYTES
}

\author{
$\mathrm{BY}$
}

\author{
A. KRZYWICKI AND T. NADZIEJA \\ Wroclaw University, Wroctaw, Poland
}

\begin{abstract}
The equations describing the density of ions which appear in the theory of electrolytes take the form $f_{t}=f_{x x}+\left(f u_{x}\right)_{x}, u_{x x}=-f$, in the one-dimensional case. In the paper the existence of solutions and their behaviour as time goes to infinity is discussed.
\end{abstract}

Consider an electrolyte consisting of identical ions which move under the influence of mutual interactions and which are contained in a subdomain $\Omega$ of $\mathbb{R}^{3}$. The temporal development of the charge density $f(t, x)$ and of the potential of electric field $u(t, x)$ is described by the following system of equations (see [1]):

$$
\begin{gathered}
f_{t}=\Delta f+\operatorname{div}(f \nabla u), \\
\Delta u=-4 \pi f .
\end{gathered}
$$

For simplicity we put all physical constants equal to one.

One usually assumes the boundary conditions

$$
\left.(\nabla f+f \nabla u) \nu\right|_{\partial \Omega}=0,
$$

where $\nu$ denotes the exterior normal of $\partial \Omega$,

$$
\left.u\right|_{\partial \Omega}=0,
$$

and the initial condition

$$
f(0, x)=f_{0}(x)
$$

Condition (3) implies that the total charge $\gamma=\int_{\Omega} f$ of ions in $\Omega$ remains unchanged for all $t \geq 0$.

The physicists claim that as time $t$ goes to infinity, $f$ and $u$ go to the stationary states $f_{\infty}$ and $u_{\infty}$, respectively. Moreover, $f_{\infty}$ has the Boltzmann form, $f_{\infty}=$ $\exp \left(-u_{\infty}\right)$ with $u_{\infty}$ satisfying the Poisson-Boltzmann equation

$$
\Delta u_{\infty}=-\exp \left(-u_{\infty}\right)
$$

and $\int_{\Omega} \exp \left(-u_{\infty}\right)=\gamma$.

Received August 21, 1990.

1991 Mathematics Subject Classification. Primary 35Q53.

Key words and phrases. System of parabolic-elliptic equations, existence of solutions, electrolytes.

(C) 1992 Brown University 
We will prove all these facts by elementary methods in the one-dimensional case when Eqs. (1) and (2) take the form

$$
\begin{gathered}
f_{t}=f_{x x}+\left(f u_{x}\right)_{x}, \\
u_{x x}=-f .
\end{gathered}
$$

To make our problem as simple as possible we take $\Omega=(-1,1)$ and assume that $f_{0}$ in $(5)$ is an even function. The invariance of Eqs. (7), (8) with respect to the transformation $x \rightarrow-x$ allows us to infer that the solutions $f, u$ also have this property and thus we can replace $(-1,1)$ by $(0,1)$ with boundary conditions

$$
\begin{gathered}
u_{x}(t, 0)=0, \quad f_{x}(t, 0)=0, \\
u(t, 1)=0, \\
f_{x}(t, 1)+f(t, 1) u_{x}(t, 1)=0,
\end{gathered}
$$

and the initial condition

$$
f(0, x)=f_{0}(x) .
$$

Note that using (8) and (9) we can replace $u_{x}(t, 1)$ appearing in (11) by $-\int_{0}^{1} f(t, y) d y$. We assume that $f_{0}$ is smooth, positive, and satisfies the compatibility condition $f_{x}(0)=0, f_{x}(1)-f_{0}(1) \int_{0}^{1} f_{0}(s) d s=0$ necessary for the regularity of $f$, which is assumed to be a classical solution of (7)-(12).

TheOREM. The problem (7)-(12) has a unique solution $f(t, x), u(t, x), u(t, x) \rightarrow$ $u_{\infty}(x), f(t, x) \rightarrow \exp \left(-u_{\infty}(x)\right)$ as $t$ goes to $\infty$ and $u_{\infty}$ satisfies $\left(u_{\infty}\right)^{\prime \prime}=$ $-\exp \left(-u_{\infty}\right)$. Moreover, $\int_{0}^{1} \exp \left(-u_{\infty}\right)=\int_{0}^{1} f_{0}$.

Proof. Condition (11) implies that if $f$ is a solution of our problem, then $\int_{0}^{1} f=$ $\sigma=$ const. This fact will be used below. Put $Q(t, x)=\int_{0}^{x} f(t, s) d s$. It is easy to verify that $Q$ satisfies Burgers' equation

$$
Q_{t}=Q_{x x}-Q Q_{x}
$$

and the relations

$$
Q(t, 0)=0, \quad Q(t, 1)=\sigma, \quad Q_{0}(x)=Q(0, x)=\int_{0}^{x} f_{0}(s) d s .
$$

Let us introduce a new function $v$ given by the formula

$$
v(t, x)=\exp \left(-\frac{1}{2} \int_{0}^{x} Q(t, s) d s-\frac{1}{2} \int_{0}^{t} Q_{x}(\tau, 0) d \tau\right),
$$

which is a slight modification of a transformation due to E. Hopf. Proceeding as usual one shows that the function $v$ is a solution of the problem

$$
\begin{aligned}
& v_{t}=v_{x x}, \\
& v_{x}(t, 0)=0, \quad v_{x}(t, 1)+\frac{\sigma}{2} v(t, 1)=0, \\
& v(0, x)=\exp \left(-\frac{1}{2} \int_{0}^{x} Q_{0}(s) d s\right) .
\end{aligned}
$$

We also have $Q=-2 v_{x} / v$. Therefore $Q$ is a solution of (13), (14) if $v>0$. From the last relation in (15) our supposition holds true if the argument $t$ is sufficiently 
small, say $t<t_{0}$. Suppose that $v\left(t_{0}, x_{0}\right)=0$ for some $x_{0} \in[0,1]$. The case $x_{0} \in(0,1)$ would contradict the strong maximum principle, and the cases $x_{0}=0$ or $x_{0}=1$ would contradict Hopf's lemma [2]; therefore $v>0$. A similar application of the maximum principle shows that $v_{x} \leq 0$, hence $Q \geq 0$. To show that $f=Q_{x}>0$ we first note that the equation satisfied by $f$ is of the form $f_{t}=a f_{x x}+b f$ with $a, b$ continuous; therefore the same arguments as before may be applied [2].

The standard Fourier method allows us to represent the solution $v$ in the form $v(t, x)=\sum_{n=1}^{\infty} a_{n} \exp \left(-\lambda_{n}^{2} t\right) \cos \left(\lambda_{n} x\right)$, where the $\lambda_{n}$ are positive solutions of the equation $\lambda=\frac{\sigma}{2} \operatorname{cotan} \lambda$.

Now

$$
-\frac{1}{2} Q=\frac{v}{v_{x}}=-\frac{\sum_{n=1}^{\infty} a_{n} \exp \left(-\lambda_{n}^{2} t\right) \lambda_{n} \sin \left(\lambda_{n} x\right)}{\sum_{n=1}^{\infty} a_{n} \exp \left(-\lambda_{n}^{2} t\right) \cos \left(\lambda_{n} x\right)},
$$

hence $\lim _{t \rightarrow \infty} Q(t, x) \rightarrow 2 \lambda_{1} \tan \lambda_{1} x$ due to $a_{1}>0$. In a similar way we find $\lim _{t \rightarrow \infty} f(t, x)=\lim _{t \rightarrow \infty} Q_{x}(t, x)=f_{\infty}(x)$, where $f_{\infty}(x)=2 \lambda_{1}^{2} / \cos \lambda_{1} x$. It is easy to verify that the function $u_{\infty}(x)=-\ln f_{\infty}(x)$ satisfies the equation $u^{\prime \prime}=-\exp (-u)$ with $\int_{0}^{1} \exp (-u)=\sigma$, which finishes the proof.

\section{REFERENCES}

[1] P. Debye and E. Hückel, Zur Theorie der Electrolyte. II, Phys. Zft. 24, 305-325 (1923)

[2] A. Friedman, Partial Differential Equations of Parabolic Type, Prentice-Hall, Englewood Cliffs, N.J., 1964 